\title{
Study of Dosage-Dependent Effects of Cytostatic Drugs Using a Fibroblast Cell Culture of the Human Nasal Mucosa
}

\author{
Vasily Yartsev ${ }^{10}$ Anna Gabashvili²,3 \\ ${ }^{1}$ Department of Lacrimal Pathology, Scientific Research Institute of \\ Eye Diseases, Moscow, Russian Federation \\ ${ }^{2}$ Fundamental Laboratory, Scientific Research Institute of Eye \\ Diseases, Moscow, Russian Federation \\ ${ }^{3}$ Cell Laboratory, Naucno-issledovatel'skij Institut Biomedicinskoj \\ Himii Imeni V N Orehovica, Moscow, Russian Federation \\ ${ }^{4}$ Cell Laboratory, Serbsky Federal Medical Research Centre for \\ Psychiatry and Narcology, Moscow, Russian Federation \\ ${ }^{5}$ Cell Laboratory, Scientific Research Institute of Eye Diseases, \\ Moscow, Russian Federation
}

Int Arch Otorhinolaryngol 2020;24:e206-e210.

\begin{abstract}
Keywords

- fibroblasts

- fibrosis

- mitomycin C

- doxorubicin

- 5-fluorouracil

- cytostatic drugs

Introduction Knowing a concentration at which cytostatic drugs are toxic for the nasal fibroblasts will enable the use cytostatic drugs in the clinical practice to prevent excessive cicatrization.

Objective To determine the cytostatic concentrations of mitomycin C, doxorubicin, and 5 -fluorouracil affecting nasal mucosa fibroblasts.

Methods We obtained material during an endonasal dacryocystorhinostomy with the patient's informed consent. The cells were cultivated. Second- to fourth-passage cells were used in the experiments. The cells were stained for vimentin and cluster of differentiation 90 (CD90). An MTS test 3 (3-(4,5-dimethylthiazole-2-yl)-5-(3-carboxymethoxyphenyl)-2-(4-sulfophenyl)-2H-tetrazolium); cell viability test was performed.

Results The cytostatic drugs have a toxic effect on cultivated fibroblasts of the nasal mucosa. This effect is dose-dependent. In terms of reducing the level of tissue fibrotisation in the nasal cavity, the most justified approach is to carry out an experimental study of the effect of mitomycin C, doxorubicin, and 5 -fluorouracil at the concentrations of $0.25 \mathrm{mg} / \mathrm{ml}, 0.25 \mathrm{mg} / \mathrm{ml}$, and $12.5 \mathrm{mg} / \mathrm{ml}$ respectively.

Conclusion The authors argue that it is inappropriate to use these cytostatic drugs to conduct studies with the goal of analyzing their antifibrotic effect on the nasal mucosa at concentrations that are either lower or higher than the aforementioned ones.
\end{abstract}

\section{Introduction}

Dacryocystorhinostomy is one of the most widespread surgeries performed on patients with primary acquired nasolacrimal duct obstruction. The purpose of this surgical intervention is to form stable epithelized anastomosis (ostium) between the lacrimal sac cavity and the nasal cavity. Despite improvements in the surgical technique, data show that the rate of recurrences of this intervention is as high as 13 to $17 \%{ }^{1,2}$ One of the most common causes for a negative surgery outcome is excessive cicatrization at the ostium site. ${ }^{3,4}$ Recently, cytostatic drugs, which are administered either applicationally or by injection into the mucous membrane of the nasal cavity or lacrimal sac at the final stage of the surgery, have become relatively received

November 26, 2018

accepted

August 19, 2019
DOI https://doi.org/

10.1055/s-0039-1697996. ISSN $1809-9777$.
Copyright (e 2020 by Thieme Revinter

Publicações Ltda, Rio de Janeiro, Brazil
License terms

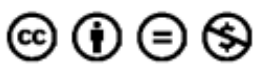


widespread to reduce the severity of the fibrotization. Cytostatic drugs are known to reduce fibroblast proliferation, which, in turn, causes a reduction in fibrotization in the region in which a cytostatic drug is administered. ${ }^{5}$

Several authors have reported an accentuated decrease in the number of lacrimal passage obliteration recurrences following the administration of cytostatic drugs, ${ }^{6,7}$ while other authors did not report this effect. ${ }^{8,9}$ The authors of the present study argue that the absence of clinically-significant fibrotization inhibition was due to the fact that the required cytostatic concentration had not yet been achieved in the tissues of the formed anastomosis region. ${ }^{8}$ The present paper is a study on the dosage-dependent effect that cytostatic drugs have on cultivated fibroblasts of the human nasal mucosa. Knowing a concentration at which cytostatic drugs retain their toxicity against nasal mucosa fibroblasts will enable the implementation of these techniques in the clinical practice to prevent excessive cicatrization when performing various surgical interventions in the nasal cavity, particularly dacryocystorhinostomy.

The purpose of the present paper is to determine the cytotoxic concentrations of mitomycin $\mathrm{C}$, doxorubicin and 5-fluorouracil that affect the nasal mucosa fibroblasts.

\section{Methods}

\section{Isolation and Characterization of the Cell Culture}

The authors obtained the histological material necessary for the study during an endonasal endoscopic dacryocystorhinostomy. Following nasal mucosa decongestion with $0.1 \%$ xylometazoline solution and local topical anesthesia with 10\% lidocaine solution (Pharmstandard-Leksredstva, Kursk, Kursk Oblast, Russia), a 1.5-mm deep horizontal incision (using a diamond-shaped knife with an incision depth limitation) was made in the lacrimal fossa projection region, and $4 \times 2$-mm section of mucous membrane was bluntly separated at that depth. This biopsy specimen was soaked in $0.04 \%$ gentacimin solution (Dalhimfarm, Khabarovsk, Khabarovskiy kray, Russia) for 30 minutes, and then transferred to the cell technology laboratory for further treatment.

The specimen was sliced into $1 \times 1-\mathrm{mm}$ fragments using a surgical scalpel. The fragments were placed in Petri dishes with a diameter and growth surface area of $3.5 \mathrm{~cm}$ and $10 \mathrm{~cm}^{2}$ respectively (Corning, Corning, NY, US) and cultured in Dulbecco's Modified Eagle's Medium (DMEM), supplemented with $2 \mathrm{mM}$ of glutamine, $100 \mathrm{U} / \mathrm{ml}$ of penicillin, $0.1 \mathrm{mg} / \mathrm{ml}$ of streptomycin (Gibco, Thermo Fisher, Waltham, MA, US) and $10 \%$ Fetal bovine serum (FBS) at $37^{\circ} \mathrm{C}$, in a humidified atmosphere containing $5 \%$ of $\mathrm{CO}_{2}$. On the 4 th to 5 th days of cultivation, the cells started migrating from the fragment tissue to the plastic. By the 14th day of cultivation, the explants were removed from the dishes, and the remaining cells were dissociated with a $0.05 \%$ Trypsin-ethylenediaminetetraacetic acid (EDTA) solution (Gibco) and passaged in T25 culture flask. Our previous experience with explant cultivation showed that the proliferation of first-passage fibroblasts is not sufficiently active. Due to this, a growth factor was added to the first passage medium. First-passage cells were cultivated in DMEM using the aforementioned composition and fibroblast growth factors (FGF, SigmaAldrich, St. Louis, MO, US) with a concentration of $4 \mathrm{ng} / \mathrm{ml}$, while the second-passage cells had no FGF treatment. The growth medium was replaced every 3 to 4 days. The cells were subcultured at a 1:4 to $1: 6$ ratio. Cell-growth monitoring and the morphology assessment were performed using a Zeiss Axio Vert.A1 (Carl Zeiss, Oberkochen, Germany) inverted microscope. Second- to fourth-passage cells were used in the experiments.

\section{Immunocytochemical Analysis}

To characterize the cell culture obtained, the cells were stained for fibroblast specific markers: vimentin and cluster of differentiation 90 (CD90). The cells were grown in Petri dishes for confocal microscopy $\left(5 \times 10^{3}\right.$ cells per dish). After attaining $50 \%$ of confluence, the cells were fixed with $4 \%$ paraformaldehyde ( 10 minutes at $4^{\circ} \mathrm{C}$ ), washed 3 times with Phosphate buffered saline (PBS), and incubated for 30 minutes at room temperature in PBS containing $0.2 \%$ tween-20, $0.2 \%$ triton $x-100$, and $2 \%$ goat serum. Then the samples were incubated with primary antibodies to vimentin (1:40; Abcam, Cambridge, United Kingdom,) in PBS with $0.2 \%$ tween-20 and $0.2 \%$ goat serum (for 1 hour at $37^{\circ} \mathrm{C}$ ), washed 3 times with PBS, and incubated with second antimouse immunoglobulin antibodies (goat anti-mouse Alexa Fluor 555, $1 \mu \mathrm{g} / \mathrm{ml}$, Invitrogen, Carlsbad, CA, US) for 1 hour at $37^{\circ} \mathrm{C}$. The samples were washed with PBS, and the cell nuclei were poststained with 4',6-diamidino-2-phenylindole (DAPI) (1:400; Invitrogen). For CD90 visualization, the cells were incubated for 10 minutes at $4^{\circ} \mathrm{C}$ with anti-CD90 primarily labeled antibodies (1:11; Miltenyi Biotec, Bergisch Gladbach, North Rhine-Westphalia, Gernamy) carrying phycoerythrin fluorescent dye (-Fig.1).

\section{Confocal Laser Scanning Microscopy}

The scanning was performed using a A1R MP+ confocal laser scanning microscope (Nikon, Shinagawa, Tokyo, Japan). The 405-nm and 561-nm emission lasers and the following optics were used in the present study: Plan Apo 20x/0,75 Dic N, Apo IR 60x/1,27 WI and Apo TIRF 60x/1,49 oil Dic lens (Nikon, Shinagawa, Tokyo, Japan). The cell contours were visualized using differential interference contrast. The images obtained were processed using the NIS-Elements AR software (Nikon).

\section{Cell Viability Assay}

the CellTiter 96 AQueous One Solution Reagent kit (Promega, Madison, WI, US) was used for the MTS test. The MTS reagent was defrosted immediately before use. The test was performed according to manufacturer's instructions (procedure TB245). The cells were cultured in 96-well plates (3,000 cells/ well) in DMEM containing 10\% FBS, antibiotics (100 units/mL of penicillin, $100 \mu \mathrm{g} / \mathrm{ml}$ of streptomycin, Gibco), and GlutaMax (2 mM, Gibco). After 24 hours of cultivation, drugs at various concentrations obtained by serial dilutions in the growth medium were added inside the wells. After 24 hours of incubation, $20 \mu \mathrm{l}$ of MTS (3-(4,5-dimethylthiazole-2-yl)-5(3-carboxymethoxyphenyl)-2-(4-sulfophenyl)-2H- 


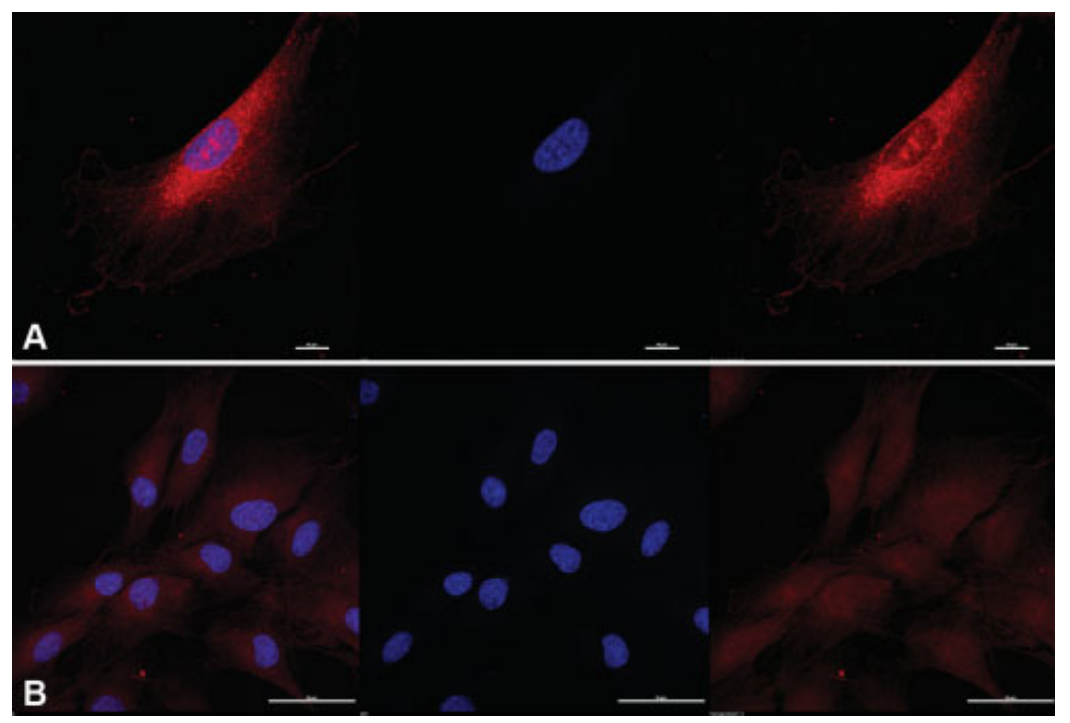

Fig. 1 Immunocytochemical staining. Confocal laser scanning microscopy (Nikon A1R MP +). Cell nuclei are stained with DAPI (1:400; Invitrogen) (blue fluorescence). Bar scale: $50 \mu \mathrm{m}$. (A) Anti-vimentin primary monoclonal antibodies (1:40; Abcam) and antimouse immunoglobulin secondary antibodies, conjugated with Alexa555 (1:750; Invitrogen) fluorescent dye (red fluorescence). (B) Primarily labeled anti-cluster of differentiation 90 - phycoerythin antibodies (1:11; Miltenyi Biotec) for CD90 visualization (red fluorescence).

tetrazolium) reagent were added to each well containing $100 \mu \mathrm{l}$ of growth medium. The cells incubated with the culture medium were used as the negative control. The culture medium without cells was used for the blank.

The optical density was measured at $490 \mathrm{~nm}$ after 4 hours of incubation with the MTS reagent. The cell viability was calculated using the following formula:

Number of living cells $=\left(A_{s}-A_{b} / A_{c}-A_{b}\right) \times 100 \%$

$A_{s}$ - mean value of the specimen's optical density (OD); mean OD sample;

$A_{b}$ - mean value of the blank sample's optical density; mean OD control blank;

$A_{c}$ - mean value of the control's optical density; mean OD control.

Values of IC50 were found with Hill equation using the GraphPad Prism 6 (GraphPad Software, San Diego, CA, US) software.

\section{Results}

As a result of the study, a human nasal mucosa fibroblast cell culture positive for specific markers (vimentin and CD90) was obtained.

The culture was used as a test system to determine the cytotoxicity effect of the following cytostatic drugs: mitomycin c, doxorubicin and 5-fluorouracil. The MTS test is a colorimetric method that enables the determination of the number of viable cells when studying cell proliferation and the cytotoxicity of various drugs. Mitochondrial NADH nicotinamide adenine dinucleotide (reduced)-dependent oxidoreductases are capable of reducing the MTS reagent to formazan, whose absorption rate reaches its maximum value at $490 \mathrm{~nm}$ to $500 \mathrm{~nm}$.

- Table 1 shows the results of the analysis of the number of viable fibroblasts depending on the concentration of the cytostatic drugs used. These data are shown in - Fig. 2. The
IC50 values for mitomycin c, doxorubicin and 5-fluorouracil were $113 \mathrm{mkg} / \mathrm{ml}, 392 \mathrm{mkg} / \mathrm{ml}$ and $19 \mathrm{mkg} / \mathrm{ml}$ respectively.

\section{Discussion}

The present paper is a study on the effect of cytostatic drugs on cultivated fibroblasts of the nasal mucosa. The cytostatic drugs studied are known to reduce fibroblast proliferation by inhibiting DNA replication. The effect of doxorubicin is intercalation, that is, integration of the nitrogen bases of the DNA, which inhibits the effect of topoisomerase II, thus making the relaxation of super-spiralized DNA sections impossible, and disturbing the transcription process. The effect of 5-fluororacil implies replacing uracil with fluorouracil in a replicated RNA molecule, which make its further processes impossible. The effect of mitomycin C leads to the formation of covalent bonds between complimentary DNA strands, thus hampering its replication.

The use of mitomycin $C$ for antifibrotic purposes has significantly increased in the lacrimal surgery practice. ${ }^{5-9}$ There are also several reports on the use of 5-fluorouracil as an antifibrotic agent for dacryocystorhinostomy. ${ }^{10-12}$ The authors found no evidence of the use of doxorubicin to prevent dacryocystitis recurrence. However, in vitro studies regarding mucosal cells showed the effect of doxorubicin on collagenogenesis. ${ }^{13}$

As the analysis of the results of the present study showed, the toxic effect that the drugs in question had on the cultivated fibroblasts of the human nasal mucosa was dosage-dependent.

The present study showed that the toxic effect that mitomycin $C$ had on fibroblasts of the human nasal mucosa was sufficient for to terminate fibroblast growth at a concentration of $0.25 \mathrm{mg} / \mathrm{ml}$, which corresponds to the data of a previous research. ${ }^{5}$

To date, no studies on the toxicity of doxorubicin and 5fluorouracil against fibroblast cultures of the nasal mucosa have been performed. In the present study, the authors found 


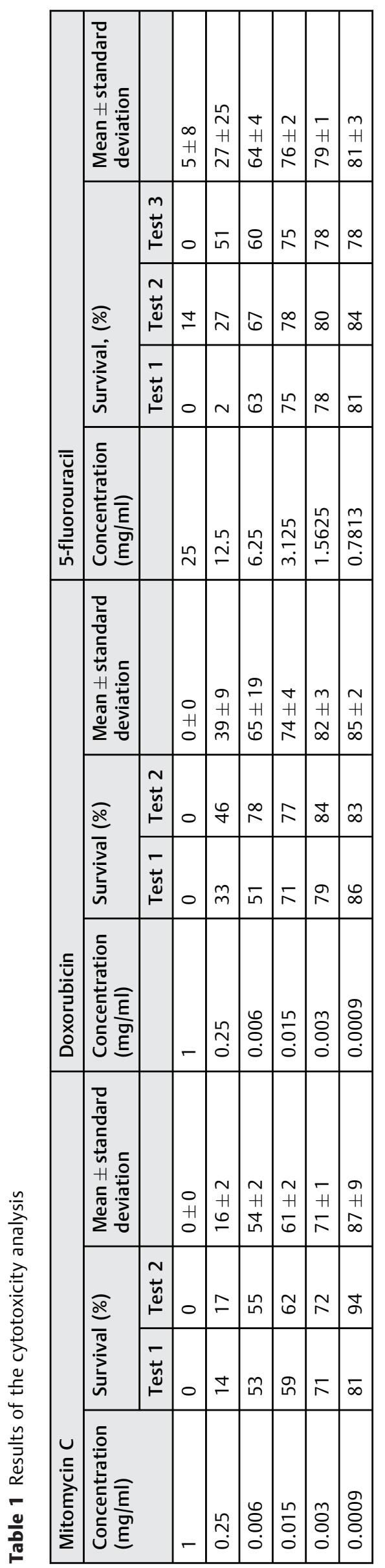

that the optimal effective doxorubicin concentration for the termination of fibroblast growth is $0.25 \mathrm{mg} / \mathrm{ml}$; yet, at this concentration, its toxic effect on the fibroblasts is smaller than that of mitomycin $C$ at the same concentration. Thus, it is plausible to expect a lower clinical effect of doxorubicin at the concentration of $0.25 \mathrm{mg} / \mathrm{ml}$, when compared to that of mitomycin $\mathrm{C}$ at the same concentration and dosage.

The analysis of the toxic effect of 5-fluorouracil showed that $12.5 \mathrm{mg} / \mathrm{ml}$ is the optimal concentration of this drug to terminate fibroblast growth; however, at this concentration its toxic effect on nasal cavity fibroblasts is also lower than that of mitomycin C when used at the concentration of $0.25 \mathrm{mg} / \mathrm{ml}$.

In all cases, an increase in concentration made the toxicity of the drugs studied get close to its absolute value, while a decrease inhibited the cell's viability to a degree apparently insufficient for the development of the clinical effects.

Surgeons currently tend to use cytostatic drugs at empirically chosen concentrations, which leads to negative outcomes and deviation from the technique: for example, in the studies by Bakri et $\mathrm{al}^{11}$ and Watts et al, ${ }^{12}$ the authors used 5fluorouracil at concentrations of $0.5 \mathrm{mg} / \mathrm{ml}$ and $25 \mathrm{mg} / \mathrm{ml}$ respectively. The present study provides a clue to explain the reason behind the lack of effect of the drug in the former study and the relatively poor results in the latter one.

The results obtained in the present study enable us to assume that the use of cytostatic drugs at exactly the concentrations determined by the authors may enable the achievement of the maximum antifibrotic effect, with a simultaneous reduction in the number of undesirable side effects on the cells, which also affects postdacryocystorhinostomy tissue regeneration processes. The authors argue that it is appropriate to use these data when carrying out experiments using a more complex model. Regarding the fact that the in vitro research could not be directly extrapolated to the clinical practice, we suppose that an experimental trial with an animal model should be an appropriate following step for the present research. The data obtained with an animal model-based research could be translated into the clinical practice.

\section{Conclusion}

The cytostatic drugs studied have a toxic effect on cultivated fibroblasts of the nasal mucosa. The authors showed that this effect is dosage-dependent. In terms of reducing the level of tissue fibrotization in the nasal cavity and, particularly, in the dacryocystorhinostomy-formed junction site, the most justified approach is to carry out an experimental study on the effect of mitomycin C, doxorubicin and 5-fluorouracil at the concentrations of $0.25 \mathrm{mg} / \mathrm{ml}, 0.25 \mathrm{mg} / \mathrm{ml}$, and $12.5 \mathrm{mg} / \mathrm{ml}$ respectively.

The authors suppose it is inappropriate to use these cytostatic drugs to conduct studies with the goal of analyzing their antifibrotic effect on the nasal mucosa at concentrations that differ from the aforementioned ones, since at such concentrations the drugs either cannot have a clinicallysignificant effect or, as the authors assume, can have an undesirable effect by inhibiting the proliferation not only of fibroblasts, but also of other cells affecting nasal mucosa reparation. 


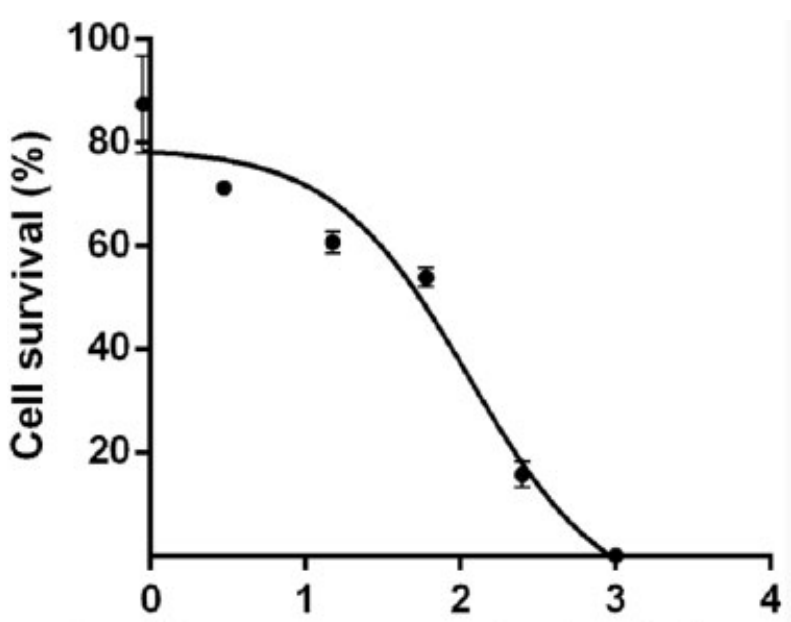

A Log[Drug concentration] ug/ml

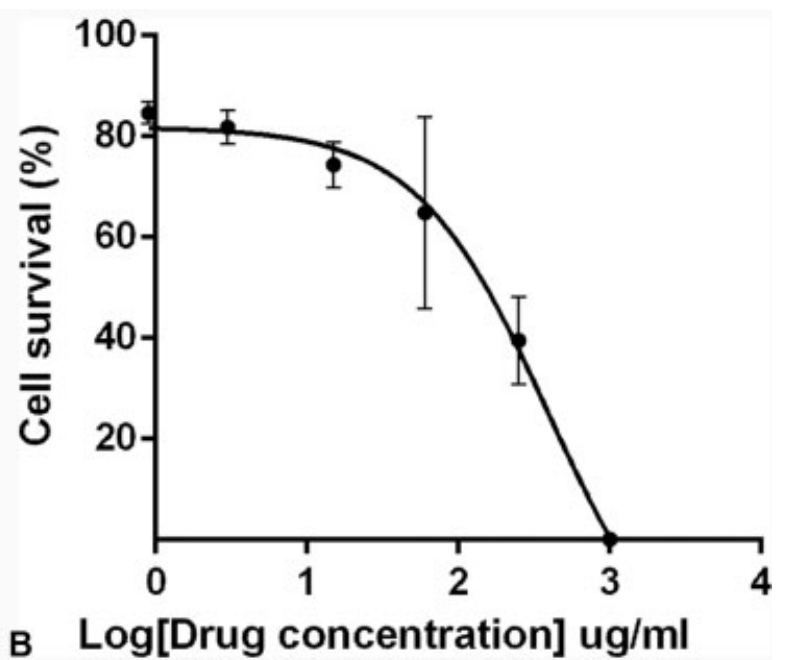

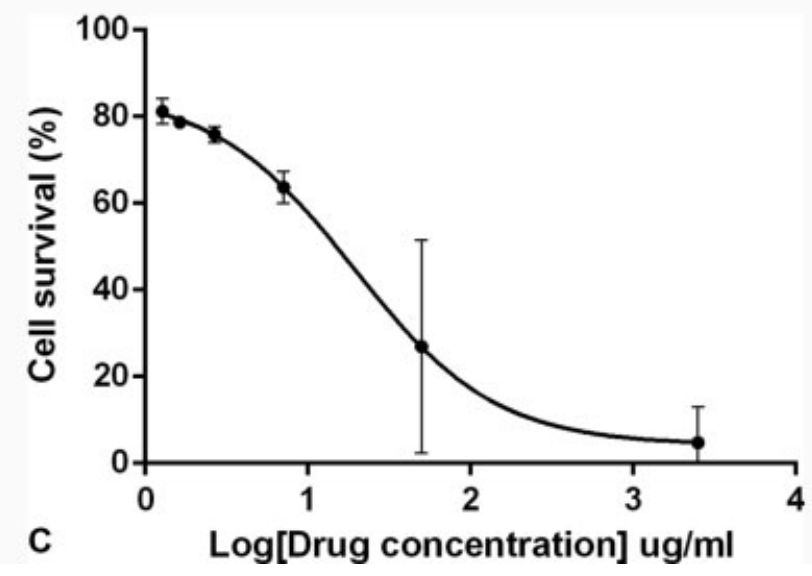

Fig. 2 Cell viability and drug concentration. (A) Mitomycin C; (B) doxorubicin; (C) 5-fluorouracil.

Conflict of Interest

The authors have none to declare.

All procedures performed in the present study were in accordance with the ethical standards of the institutional and national research committee and with the 1964 Helsinki declaration and its amendments. The present research was approved by the local Ethical Committee of Scientific Research of the Institute of Eye Diseases.

\section{References}

1 Huang J, Malek J, Chin D, et al. Systematic review and meta-analysis on outcomes for endoscopic versus external dacryocystorhinostomy. Orbit 2014;33(02):81-90. Doi: 10.3109/01676830.2013.842253

2 Syed MI, Head EJ, Madurska M, Hendry J, Erikitola OC, Cain AJ. Endoscopic primary dacryocystorhinostomy: are silicone tubes needed? Our experience in sixty three patients. Clin Otolaryngol 2013;38(05):406-410. Doi: 10.1111/coa.12152

3 Dave TV, Mohammed FA, Ali MJ, Naik MN. Etiologic analysis of 100 anatomically failed dacryocystorhinostomies. Clin Ophthalmol 2016;10:1419-1422. Doi: 10.2147/OPTH.S113733

4 Lin GC, Brook CD, Hatton MP, Metson R. Causes of dacryocystorhinostomy failure: External versus endoscopic approach. Am J Rhinol Allergy 2017;31(03):181-185. Doi: 10.2500/ajra.2017.31.4425

5 Ali MJ, Mariappan I, Maddileti S, Ali MH, Naik MN. Mitomycin C in dacryocystorhinostomy: the search for the right concentration and duration-a fundamental study on human nasal mucosa fibroblasts. Ophthal Plast Reconstr Surg 2013;29(06):469-474. Doi: 10.1097/IOP.0b013e3182a23086

6 Jawad M, Ali Z, Tariq S, Qayum I, Aftab H. Effect of Intraoperative Mitomycin-C Application in Outcome of External Dacryocystorhinostomy. J Ayub Med Coll Abbottabad 2015;27(03):598-600

7 Mukhtar SA, Jamil AZ, Ali Z. Efficacy of external dacryocystorhinostomy (DCR) with and without mitomycin-C in chronic dacryocystitis. J Coll Physicians Surg Pak 2014;24(10):732-735. Doi: 10.2014/JCPSP.732735

8 Atkova EL, Fedorov AA, Root AO, Iartsev SD, Krakhovetsky NN, Yartsev VD. Causes of unsatisfactory results of the use of mitomycin-C in endoscopic endonasal dacryocystorhinostomy. Saudi J Ophthalmol 2017;31(03):150-155. Doi: 10.1016/j.sjopt.2017.05.007

9 Ozsutcu M, Balci O, Tanriverdi C, Demirci G. Efficacy of adjunctive mitomycin $\mathrm{C}$ in transcanalicular diode laser dacryocystorhinostomy. Eur Arch Otorhinolaryngol 2017;274(02):873-877. Doi: 10.1007/ s00405-016-4308-7

10 Abraham LM, Selva D, Casson R, Leibovitch I. The clinical applications of fluorouracil in ophthalmic practice. Drugs 2007;67(02):237-255

11 Bakri K, Jones NS, Downes R, Sadiq SA. Intraoperative fluorouracil in endonasal laser dacryocystorhinostomy. Arch Otolaryngol Head Neck Surg 2003;129(02):233-235

12 Watts P, Ram AR, Nair R, Williams H. Comparison of external dacryocystorhinostomy and 5-fluorouracil augmented endonasal laser dacryocystorhinostomy. A retrospective review. Indian J Ophthalmol 2001;49(03):169-172

13 Sakagami H, Okudaira N, Masuda Y, et al. Induction of Apoptosis in Human Oral Keratinocyte by Doxorubicin. Anticancer Res 2017; 37(03):1023-1029. Doi: 10.21873/anticanres.11412 